\title{
L'HOMME L'Homme
}

157 | janvier-mars 2001

Représentations et temporalités

\section{Représentations de la société, statuts et temporalités à Arama (Nouvelle-Calédonie)}

Denis Monnerie

\section{OpenEdition}

Journals

Édition électronique

URL : http://journals.openedition.org//homme/96

DOI : 10.4000/lhomme.96

ISSN : 1953-8103

Éditeur

Éditions de l'EHESS

Édition imprimée

Date de publication : 1 janvier 2001

Pagination : 59-86

ISBN : 2-7132-1357-6

ISSN : 0439-4216

Référence électronique

Denis Monnerie, «Représentations de la société, statuts et temporalités à Arama (Nouvelle-

Calédonie) », L'Homme [En ligne], 157 | janvier-mars 2001, mis en ligne le 23 mai 2007, consulté le 20

avril 2019. URL : http://journals.openedition.org/lhomme/96 ; DOI : 10.4000/lhomme.96 


\title{
Représentations de la société, statuts et temporalités à Arama
}

(Nouvelle-Calédonie)

\author{
Denis Monnerie \\ À la mémoire de Louis Dumont \\ "Le concept de "temps" se rapporte tantôt à une séquence continue de chan- \\ gements non répétables et tantôt à des séquences répétables de changement." \\ Norbert Elias
}

LES PRINCIPES fondamentaux de l'organisation des sociétés kanak de l'extrême nord de la Grande Terre restent aujourd'hui encore, pour une large part, énigmatiques. Après James Cook, le découvreur, les premiers observateurs européens - prêtres catholiques et militaires français - ont remarqué l'existence de personnages importants, qu'ils nommèrent " chefs ", ou " grands chefs ». Or, bien vite la culture européenne de ces prêtres et militaires disciplinés vint buter, ici comme ailleurs en Océanie, sur l'incompréhension du rôle véritable de ceux qu'ils avaient ainsi qualifiés. Car ces termes impliquaient des relations de commandement qui ne sont pas des prérogatives de ces personnages - ou alors de façon épisodique. Par ailleurs, eux-mêmes impliqués dans des opérations missionnaires ou coloniales et soucieux de se ménager des alliés dans la population autochtone, ces observateurs assistèrent à des regroupements guerriers qui associaient assez systématiquement " chefferies » Hoot contre « chefferies » Whaap. Il existait donc, au-delà des " chefferies ", une organisation d'emprise régionale, connue sous le nom de Hoot ma Whaap (Douglas 1970 ; Guiart 1966 ; Lambert 1900).

Avant tout je voudrais remercier pour leur amitié, leur hospitalité et leur collaboration tous mes hôtes kanak d'Arama et de Nouvelle-Calédonie, hommes et femmes de tous âges, qui m’ont permis de mener à bien ce travail sur le terrain. Chacun à leur façon, ils m’ont fait partager un attachement profond à leur pays, à leur société et à leurs langues. Ce travail a été effectué en résidence à Arama (Bouarou) lors de quatre séjours d'une durée totale de plus de deux ans et demi, entre août 1992 et octobre 1998 en situation d'observation participante et, au fur et à mesure de l'apprentissage, dans la langue d'Arama, le nyelayu. Les données présentées ici ont été rassemblées lors de nombreux entretiens, réunions et cérémonies. Le financement de ces terrains a été assuré par le programme ESK (Ministère de la Culture et Ministère des DOM-TOM), l'équipe ERASME (UPR 262 du CNRS, EHESS), la mission de la Recherche du Ministère de l'Éducation nationale et la CORDET, que je remercie également. Merci enfin à tous les amis qui, à Nouméa et à Paris, de façons très diverses, ont facilité ce travail. Les publications concernant les gens d'Arama sont principalement le fait de voyageurs et missionnaires (Garnier 1978; Kling 1989; Mortelier 1993; Rozier 1990). 
La recherche sur le terrain qui est à l'origine de cet article a été centrée sur Arama et étendue aux relations dans la région Hoot ma Whaap. Arama est un village de l'extrême nord de la Grande Terre, situé en bord de mer, sur la côte est, où vivent quatre cents habitants, principalement horticulteurs, pêcheurs et éleveurs. Dans la conversation courante, pour référer à ce village et à la société, le nom d'Arama (Aramwa) est utilisé. Toutefois la société est conçue comme une Grande Maison ${ }^{1}$ nommée Teâ Aâôvaac, elle-même composée de quatre hameaux ou Maisons ${ }^{2}$. La notion de Grande Maison réfère à la fois à l'organisation sociale, à un bâtiment - grande maison ronde à toit conique - et à tous les êtres d'un territoire bordé par la mer, des cours d'eau et des lignes de crête.

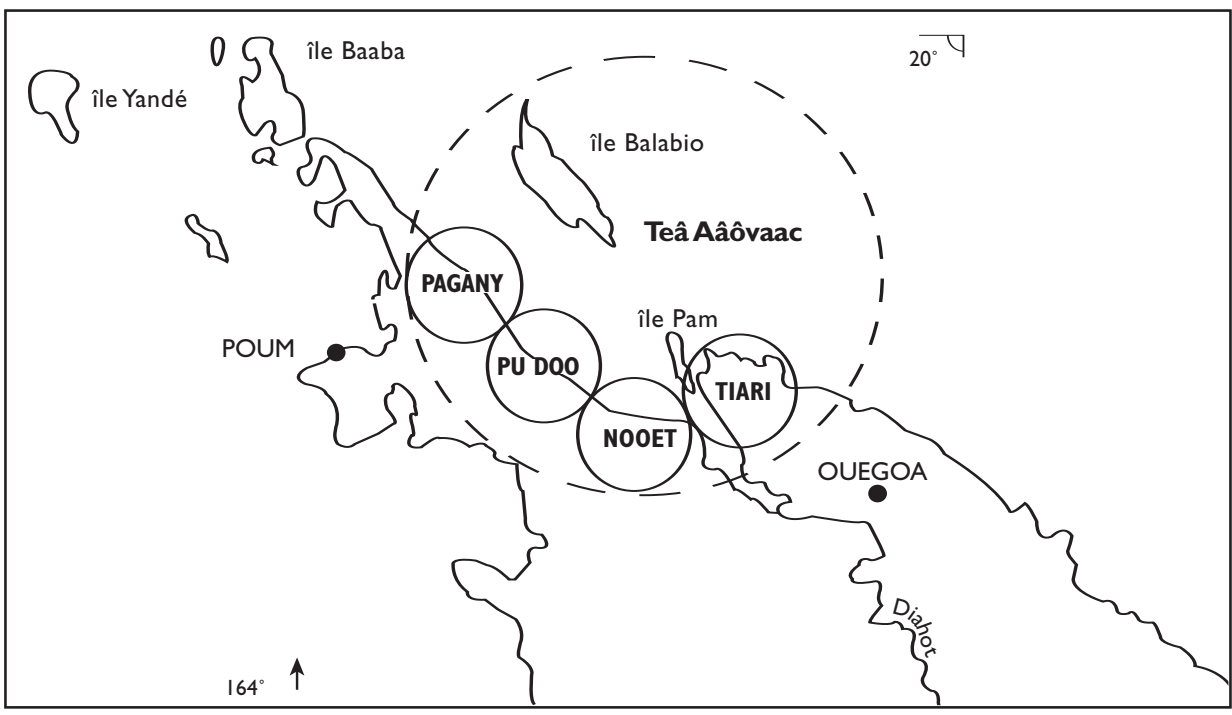

Carte d'Arama et schéma de la Grande Maison Teâ Aâôvaac et des Maisons qui la constituent

La région Hoot ma Whaap s'étend depuis Belep et l'extrême nord de la Grande Terre jusqu'à Hienghène sur la côte est et Voh sur la côte ouest. Chaque société locale y est soit Hoot soit Whaap et forme une unité dans les relations régionales. La compréhension des sociétés de cette région implique la prise en compte de leurs relations régionales. Des formes transformées, acculturées, des cérémonies qui caractérisent ces relations régionales sont utilisées avec les autres régions de Nouvelle-Calédonie, la puissance coloniale française et le monde moderne (Monnerie, à paraître).

1. Les Grandes Maisons sont généralement nommées "grandes chefferies ", parfois "chefferies ", en français calédonien.

2. Dans ce texte, maison ou grande maison réferent à des bâtiments, les majuscules aux mots Maison et Grande Maison signalent qu'ils renvoient à l'organisation sociale. 
La Grande Maison d'Arama se présente sous deux angles : celui des relations « internes » et celui des relations " externes ». La plupart des cérémonies importantes de cette société actualisent la Grande Maison à travers les statuts de ses Maisons, de leurs dignitaires et groupes importants, qui sont aussi décrits dans de grands discours cérémoniels prononcés lors des fêtes régionales. Ce rôle de premier plan tenu par les statuts des entités sociales - qui, je le montrerai, commandent l'ordre de préséance pendant les cérémonies, et l'ordre d'énonciation dans ces discours - explique pourquoi les relations de commandement qui, dès le début de la colonisation, furent au centre de la réflexion des Européens sur la Nouvelle-Calédonie, ne rendent compte que de façon partielle des sociétés kanak de Hoot ma Whaap, sans jamais atteindre les principes fondamentaux de leur organisation ${ }^{3}$.

\section{Société et maison en Nouvelle-Calédonie}

La plupart des auteurs ont relevé l'importance de la grande maison ronde, ou "grande case", en tant que construction architecturale ayant des liens étroits avec l'organisation sociale. Maurice Leenhardt (1980 : 125) généralise à partir de Houaïlou, Alban Bensa et Jean-Claude Rivierre (1982 : 37-40) montrent les difficultés d'une typologie, Alban Bensa (in Boulay 1990 : 17-19) résume les données concernant le centre de la Grande Terre. Toutefois, alors même que dans leurs travaux, ils insistent sur l'importance des conceptions concernant ces maisons, sur la localité et le «facteur résidentiel " (Bensa in Boulay, ibid. : 19), aucun de ces auteurs ne propose une présentation et une analyse détaillées d'une ethnographie particulière, précisément localisée, à partir de laquelle il serait possible de fonder la comparaison.

M. K. Ipère décrit, pour la région Xaracuu, les traits essentiels du " concept» de "grande case », exprimant «l'existence hiérarchisée de clans qui peuplent des régions étendues. En langage coutumier ${ }^{4}$ est transposée l'image de la case pour définir d'un seul coup la région », constituée "soit par une chefferie unique, soit par un partage de régions d'origine ancestrale» (FNLKS 1988). Pour Lifou, Jean Guiart (1992a : 352) écrit que «la construction de la grande case ronde de la chefferie de Hnupel [...] manifeste théoriquement la solidarité des différents appuis » de cette chefferie. Sur ce plan très général où la Grande Maison exprime des aspects importants de l'organisation de la société, on relève un facteur d'unité de l'ar-

3. Sur une autre question - la division Mélanésie-Polynésie - Nicholas Thomas (1989a) a montré comment le paradigme scientifique dominant a son origine dans les préoccupations et observations des premiers voyageurs européens (voir Sahlins 1963; Monnerie 1998).

4. Les termes " coutume » et " coutumier » sont utilisés en français calédonien pour décrire les actes, relations sociales et cérémonies kanak. 
chipel. Patrice Godin (1990) aborde pour Hienghène l'usage de termes désignant la maison et ses parties pour décrire les relations sociales.

Cet article rend compte des principes d'organisation et de représentation de la Grande Maison d'Arama. Je présenterai d'abord les grandes lignes de l'organisation de la société, conçue comme une Grande Maison. Après avoir exposé les spécificités du personnage suprême de la société, le teâma, et de sa famille, j'aborderai les statuts des quatre hameaux, nommés Maisons, et des groupes qui constituent cette Grande Maison. Puis j'analyserai un des discours formalisés qui, lors des cérémonies régionales, présentent la Grande Maison. Cela amène à mettre au jour un idiome qui décrit les relations d'aînesse et la nomination dans la famille du teâma, et définit les statuts des entités constituant la Grande Maison. Ces statuts commandent l'ordre d'énonciation dans ces discours et l'ordre de préséance des grandes cérémonies internes. Dans un premier temps, donc, je m'attacherai à décrire et comprendre au plus près de leurs spécificités les conceptions exprimées dans la langue d'Arama, les faits observés et leurs entrecroisements. Cela permettra, dans la cinquième partie, de confronter ce système kanak de Grande Maison avec des outils conceptuels de l'anthropologie proposés par Marshall Sahlins, James Fox et Louis Dumont ${ }^{5}$.

\section{La Grande Maison Teâ Aâôvaac}

La «Grande Maison» (mweemwa) Teâ Aâôvaac est constituée de quatre hameaux - aux habitations largement espacées - eux-mêmes nommés "Maisons » (mwa), classées par ordre de statut décroissant de la façon suivante : la Maison Pu Doo est teâ, les Maisons Nooet et Tiari ${ }^{6}$ sont ââ $\hat{o}$, la Maison Pagany est mweau. La Maison Pu Doo - son nom signifie « origine de la terre " - , centre cérémoniel de Teâ Aâôvaac est le lieu de résidence du teâma et de sa famille. Là, on érige la "grande maison " (mweemwa), bâtiment où se réunissent les dignitaires et devant laquelle se

5. Différentes versions, ou parties, de cet article ont été présentées dans les séminaires ou conférences suivantes: Table ronde : étude des sociétés kanak, EHESS, Paris (1995); séminaires du Groupe de travail en anthropologie sociale, Maison des sciences de l'homme, Paris (1996-1998); séminaires d'ethnologie de l'Université de Münster, Allemagne (1996); séminaire d'ethnologie de l'Université de Leyde, Pays-Bas (1997). Je remercie pour leurs critiques et suggestions Cécile Barraud, Alban Bensa, Isabelle Berdah, Stéphane Breton, Françoise Cayrol, Sophie Chave-Dartoen, Christian Godin, Daniel de Coppet, Dominique Fasquel, Michael Houseman, I. Gaudenz Domenig, André Iteanu, Jaarich Oosten, Jos Platenkamp et Françoise Ozanne-Rivierre - dont j'utilise le système de transcription du nyelayu (1998). La carte a été réalisée par Françoise Berdah.

6. Aujourd'hui, trois faits tendent à accentuer la distance sociale de Tiari vis-à-vis des trois autres Maisons. La plupart des déplacements s'effectuent par la route - très longue - alors qu'autrefois nombre de déplacements se faisaient en bateau. Tiari possède une église, un cimetière et une paroisse distincts, alors que l'église, le cimetière et la paroisse d'Arama sont communs à Pu Doo, Nooet et Pagany. Enfin Tiari est rattachée administrativement à la commune de Ouégoa alors qu'Arama fait désormais partie de la commune de Poum. L'influence de ces faits sur certaines cérémonies est discutée in Monnerie (à paraître). 
déroulent nombre des cérémonies importantes de la société 7 . Son édification et son architecture actualisent les solidarités et les statuts de la société. La notion de Grande Maison associe donc une conception de la société à une architecture, en une relation qui inclut les fonctions du bâtiment, des actes de sa construction, des éléments de sa structure et leur représentation (voir infra, pp. 69-70).

Les personnes résidant dans la Grande Maison appartiennent soit à des "groupes importants" (paabon) ${ }^{8}$, soit à des "sous-clans" (yameevu), soit à des "groupes limitrophes" (hova mwa ou hop), soit à des "groupes accueillis " récemment. Tous ces groupes sont nommés. La plupart s'assemblent par paires lors des échanges cérémoniels. Plusieurs de ces groupes peuvent être rassemblés sous le "grand nom" (naar hâc) d'un "clan" (phwâmeevu ou phwâyameevu). Chaque groupe est en relation privilégiée avec une série de charges et de compétences cérémonielles, techniques, architecturales pour la construction de la grande maison, etc., et un ou plusieurs lieux de sa Maison.

Bien que la description - complexe - de ces groupes ne soit pas le sujet de cet article, je voudrais souligner deux points. Premièrement, ils ont souvent des traditions orales de division, de déplacement, et de rattachement antérieur à d'autres lieux et d'autres groupes, dans cette Grande Maison ou ailleurs ${ }^{9}$. Ces phénomènes ont été décrits par la quasi-totalité des observateurs de la Nouvelle-Calédonie. Ils impliquent, je le souligne, de considérer procédures et relations d'accueil comme centrales pour la compréhension des sociétés kanak. Deuxièmement, décrire l'organisation de ces groupes implique de se référer aux échanges cérémoniels. Cela permet d'insister à nouveau sur l'importance de la prise en compte des actes cérémoniels pour la compréhension des diverses facettes de l'organisation de cette société (Monnerie, à paraître).

Le classement des Maisons, de leur dignitaires et groupes importants, les termes utilisés pour définir leurs statuts, leurs imbrications et implications dans les relations sociales, renvoient à plusieurs particularités du teâma - le personnage suprême de la société - et de sa "famille».

7. Dans la configuration observée de 1992 à 1998, il n’y avait pas de grande maison construite à Arama. Toutes les cérémonies et réunions concernant la Grande Maison se déroulaient à Pu Doo (à proximité de l'église ou chez l'orateur). À l'occasion de grandes cérémonies, Balade (Grande Maison Teâ Puma) a construit en 1993 une grande maison, jouxtée d'une "maison des femmes" (mwa wo), selon une configuration caractéristique d'Arama et Balade. Devant cette grande maison se sont déroulées plusieurs cérémonies d'accueil thiam (voir infra et Monnerie, à paraître).

8. Dans la parenté, paabon désigne les petits-enfants et, en fonction du système de nomination le plus général qui fonctionne sur l'alternance des générations, implique qu'ils sont des grands-parents (voir infra, p. 64). Cela est une forme rhétorique d'atténuation. Ma traduction par "groupe important " ne rend pas compte de cette nuance (voir infra, pp. 71-72).

9. Ainsi les noms des groupes ou dignitaires d'Arama peuvent se retrouver dans d'autres Grandes Maisons de la région Hoot ma Whaap, ou au-delà. 


\section{Le teâma Aâôvaac et sa famille}

L'expression française "grand chef » est souvent utilisée à tort pour traduire le mot teâma, qui se compose de teâa "aîné », et du suffixe collectif valorisant -ma. Le teâma est donc l'aîné collectif et suprême. L'aîné des garçons du teâma est teâ, son frère cadet est le mweau. Le teâ devient teâma après une cérémonie d'intronisation, qui doit faire l'objet d'un large consensus et n'est envisagée que lorsqu'il remplit certaines conditions - aujourd'hui, principalement, jouir de la considération générale et être marié et père de famille ${ }^{10}$. Seul le fils aîné du teâma est teâ, les fils aînés d'autres branches de la famille du teâma sont ââô.

Un mythe concernant le teâma d'Arama dit que celui-ci vient d'une autre région, il est capturé en forêt, ses scarifications faciales témoignent de son statut important. La société décide d'en faire son nouveau teâma. L'ancien teâma est démis, son clan "accueille» (wove) le nouveau teâma et lui donne une épouse. Le nom personnel du nouveau teâma est Koi, son frère cadet, le mweau, se nomme Pwanaan ${ }^{11}$. Ce mythe rend compte d'une relation fondatrice de la Grande Maison - relation d'accueil et d'affinité entre le clan accueillant (le phwâmeevu Teâ Yhuen) et la "famille du teâma" (bulaya aju) qui est nommée Aâovaac, tout comme la Grande Maison dans la configuration inaugurée par ce récit.

\section{Des systèmes de nomination contrastés}

Il existe pour les hommes deux systèmes d'attribution des noms personnels kanak - que j'appelle systèmes de nomination. Le système quasi général repose sur la succession de deux noms qui se répètent avec l'alternance des générations. Dans une réalisation simple de ce système, un homme portera un nom A, son fils le nom B, son petit-fils le nom A, son arrière petit-fils le nom $B$. Il y a ici retour du même nom après deux générations, selon un système périodique binaire. Dans un groupe donné, cela concerne principalement les noms personnels kanak des deux, trois ou quatre premiers garçons (parfois filles) à naître - ou à être adoptés - et permet de perpétuer des noms importants qui correspondent à des rôles, des

10. En attendant que les conditions de l'intronisation du teâma soient remplies, l'orateur de la Grande Maison - l'ancien du clan accueillant Teâ Yhuen - exerce une sorte de régence qui implique la présence à ses côtés d'un homme de la famille du teâma. C'est cette configuration qui prévalait de 1992 à 1999. 11. Je résume un mythe (vajama) recueilli auprès du clan accueillant. Il s'agit d'un modèle très répandu sur la Grande Terre, celui de l'accueil et de l'installation d'un personnage suprême venu de l'extérieur, ceci est un autre signe d'homogénéité de la Grande Terre. À dessein, pour décrire ce clan accueillant, j'évite le terme "maître de la terre" qui prête à confusion, en particulier dans le contexte foncier actuel. Enfin précisons que l'aînée et la cadette des filles du teâma, dont les noms/titres sont Kaavo et Hixe (et ce dans tout Hoot ma Whaap), sont comme les pendants féminins du teâ et du mweau. Leurs rôles relèvent d'autres plans de la société et seront étudiés dans un autre contexte. 
statuts, des savoirs, des relations à des lieux, etc., caractérisant leur groupe. Les autres enfants reçoivent des noms personnels kanak qui, bien souvent, ne seront pas repris aux générations suivantes.

Par contraste avec ce système, les noms du teâ et du teâma $(\mathrm{T})$ et de son frère cadet le mweau $(\mathrm{M})$ se répètent après trois générations, selon un système de nomination lui aussi périodique, mais ternaire.

\begin{tabular}{lcc}
\hline & $\begin{array}{c}\text { teâma } \\
(T: \text { nom du teâma })\end{array}$ & $\begin{array}{c}\text { mweau } \\
(\mathrm{M}: \text { nom du mweau })\end{array}$ \\
\hline Mythe et génération I 12 & $\mathrm{TI}:$ Koi & $\mathrm{MI}:$ Pwanaan \\
Génération 2 & T2 : Dui & $\mathrm{M}$ : Gany \\
Génération 3 & T3 : Bweon & $\mathrm{M}$ : Kaaba \\
Génération 4 & TI: Koi & MI : Pwanaan \\
& (etc.) & \\
\hline
\end{tabular}

Tabl. I Système des noms personnels du teâma et du mweau

Les noms de la première génération, qui sont ceux du mythe, sont plus valorisés que ceux de la deuxième, eux-mêmes plus valorisés que ceux de la troisième. La génération suivante réactive les noms prestigieux de la première génération. Une part de la réflexion poursuivie dans cet article a trait aux indices de valorisation qui s'attachent à ces noms et au vocabulaire de l'aînesse. Deux critères de distinction de la famille du teâma, vocabulaire des relations d'aînesse (teâ, ââ $\hat{o}$ et mweau) et système de nomination, sont mis en œuvre pour décrire l'organisation de la Grande Maison.

\section{Le statut des Maisons}

La Maison Pu Doo est teâ, la Maison Pagany mweau. Entre ces deux statuts, le terme ââô définit une position médiane pour les Maisons Nooet et Tiari. (Nous verrons plus loin comment les statuts respectifs de ces deux dernières Maisons sont déterminés en rapport avec les titres de leurs dignitaires, qui sont aussi des noms de la famille du teâma; voir infra, Tabl. 2.1 et 2.2. et p. 68). J'examinerai d'abord la façon dont ces statuts sont exprimés dans les discours des cérémonies régionales. Cela pour montrer qu'un idiome commun informe, d'une part, l'expression de l'aînesse et de la nomination dans la famille du teâma et, d'autre part, la formulation des différences de statuts dans la Grande Maison.

12. Dans la suite du texte les abréviations T1, M1, etc. seront utilisées quand il sera nécessaire de préciser la position d'un nom dans ce système et donc son indice de valorisation. 


\section{L'expression des statuts dans les discours}

Dans la région Hoot ma Whaap de grandes fêtes rassemblent plusieurs sociétés ou Grandes Maisons. La Grande Maison organisatrice accueille, tour à tour, chacune des délégations des autres Grandes Maisons dans une cérémonie nommée thiam ${ }^{13}$. Les arrivants se présentent par un discours cérémoniel rythmé nommé hoor, décrivant d'abord le «Chemin » (daan) qui les relie à leurs hôtes, puis leur société, sous une forme concise que je nomme formule hoor ${ }^{14}$. Les deux Grandes Maisons, celle qui arrive et celle qui reçoit, après un échange d'autres discours, de biens cérémoniels et de nourritures, sont alors rassemblées, provisoirement, en une seule entité cérémonielle, qui, de façon analogue, va accueillir les autres délégations participant à la fête.

Pour ces cérémonies, l'orateur d'Arama est l'ancien du clan accueillant. Dans la formule hoor, il énonce les noms de la Grande Maison, de ses Maisons, de leurs groupes et dignitaires. Les gens d'Arama venus en délégation pour faire le thiam l'approuvent, l'encouragent, par des exclamations, tout particulièrement quand le discours cite le nom de leur propre Maison ou groupe. L'expression de cette approbation scande la formule hoor et lui répond comme un dialogue.

\section{La description de la Grande Maison dans la formule hoor}

La formule hoor est une description très formalisée et condensée de la Grande Maison. Les quatre Maisons y sont présentées l'une à la suite de l'autre, dans l'ordre de leur statut décroissant par une séquence oratoire répétée quatre fois. Chaque séquence a une structure de base identique, composée de segments scandés séparés par une courte pause. Le tableau qui suit synthétise cette succession de quatre séquences oratoires (désignées par les lettres $\mathrm{A}$ à $\mathrm{D}$ ), et leur organisation en segments, numérotés de 1 à 3 , suivis d'une série de segments numérotés $4 \alpha, 4 \beta, 4 \gamma$, etc. Le premier segment de chaque séquence nomme la Maison dont l'organisation va être décrite dans les segments suivants : d'abord par les noms de ses groupes importants (seg. 2), puis par les titres de ses dignitaires (seg. 3) et, dans la série de segments qui suit (seg. $4 \alpha, 4 \beta, 4 \gamma$, etc.), par les noms des autres groupes de la Maison. À l'exception du premier, la quasi-totalité de ces segments rassemblent par paires les entités qu'ils nomment.

13. Sous la forme classique résumée ici, cette cérémonie et sa préparation ont été observées en 1993 à Bondé et Balade. On trouvera la transcription et la traduction de l'intégralité des discours tenus lors d'un de ces thiam in Monnerie (à paraitre), ainsi qu'une étude comparative de la forme classique et des formes acculturées de cette cérémonie

14. Il est parfois dit qu’un discours hoor est fait "sur le bois", en référence à la branche de l'arbre qui avec le message - constitue l'invitation formelle à la cérémonie. Dans d'autres régions l'expression « discours sur le bois» renvoie à d'autres modalités des discours (voir Ammann 1997 : 63-64). 
Avant d'analyser ces séquences, segment par segment, je voudrais insister sur le fait que je transcris et analyse une formule orale, prononcée dans une cérémonie complexe. Je ne puis que suggérer dans cet article l'importance des gestes cérémoniels, de la chorégraphie et, dans l'énonciation de cette formule, celle de la prosodie, du placement du souffle et de la synchronisation entre participants (Monnerie, à paraître). J'insisterai d'abord sur deux phénomènes fondamentaux, qui constituent l'armature de cette formule et qui "passent " bien à la transcription, à savoir l'ordre d'énonciation des séquences et des segments et le groupement par paires de la plupart des entités qui constituent la Grande Maison (et qui sont décrites dans les segments 2,3 et 4 ).

\section{Séquence A I / Teâ Aâôvaac \\ (Pu Doo)* \\ 3 / Sur les noms de Koi et Pwanaan \\ $4 \alpha / \beta / \gamma$ [etc. num ration de tous les groupes de la Maison Pu Doo]

$\begin{array}{ll}\text { Séquence B } & \text { I / Maison Nooet } \\ & 2 \text { / Paabon Dui et Paabon } \\ & \text { Waala } \\ & 3 / \text { Sur les noms de Dui } \\ & \text { et Bwae } \\ & 4 \alpha / \beta / \gamma \text { [etc. num ration de tous } \\ & \text { les groupes de la Maison Nooet] }\end{array}$ \\ Séquence C I/Maison Tiari \\ 2 / Paabon Tea et Paabon Wiya \\ 3 / Sur les noms de Bweon et Kaaba \\ $4 \alpha / \beta / \gamma$ [etc. num ration de tous \\ les groupes de la Maison Tiari] \\ Séquence D I / Maison de bord de mer Pagany \\ $2 /$ Paabon Gany et Paabon Deedan \\ 3 / Sur les noms de Gany et Damagilu \\ $4 \alpha / \beta / \gamma /$ [etc. num ration de tous les groupes de la Maison Pagany]}

\footnotetext{
* Les parenth ses $r$ f rent aux aspects implicites de la premi re s quence, tudi s plus loin
}

Tabl. 2.I La formule hoor
La formule hoor est la formalisation oratoire de rapports sociaux entre Maisons, entre dignitaires et entre groupes. Le procédé rhétorique principal en est l'ordre d'énonciation des séquences, des segments et des noms; il indique le statut des entités sociales - groupes ou personnes - constituant la Grande Maison. Simultanément, une part non négligeable de ces rapports de statut est laissée implicite et l'étude de la formule hoor implique à la fois l'analyse des procédés d'énonciation, qui suit, et celle du non-dit, qui viendra plus loin.

1) Dans chaque séquence, le premier segment présente le nom d'une Maison, dans l'ordre : Teâ Aâovaac - la Grande Maison - puis les trois Maisons Nooet, Tiari, Pagany. Soulignons que Pu Doo n'est pas nommée dans la première séquence, je reviendrai sur ce point.

2) Ce segment présente d'abord une paire de "groupes importants", le premier, Paabon X, est teâ, aîné, le second groupe, Paabon Y, est mweau, cadet. On verra pourquoi ce segment n'existe pas dans la première 
séquence - où les relations entre le clan accueillant et la famille du teâma sont laissées implicites.

3) Puis l'orateur dit: "Sur les noms de V et W», nommant une paire de dignitaires appartenant aux groupes importants qui ont été décrits dans le segment précédent. Le premier est teâ, le second est mweau. Lexpression «sur les noms de" (bwe naaran) signifie qu'un nom personnel de teâma, de mweau, ou d'hommes de la famille du teâma, est, pour ces dignitaires, un titre, désignation exprimant un statut élevé. Les deux paires présentées dans les segments 2 et 3, groupes importants et dignitaires, sont énoncées dans l'ordre de leur relation d'aîné teâ à cadet mweau, par ordre décroissant de statut.

4) Vient ensuite une série de segments ( $4 \alpha, 4 \beta, 4 \gamma$, etc.) constitués des noms de tous les sous-clans puis des groupes accueillis de la Maison, enfin de ses groupes limitrophes, qui résident au contact d'autres Grandes Maisons. Le plus souvent, ces segments sont constitués de noms groupés par paires et ils sont énoncés dans un ordre qui ne varie guère d'un discours à l'autre.

Ainsi, dans la formule hoor, dans les trois premiers segments de chaque séquence, les termes teâ et mweau dénotant les relations d'aînesse de la famille du teâma sous-tendent le classement des Maisons, des groupes importants et de leurs dignitaires. Les noms personnels du teâma, du mweau et d'autres hommes de leur famille deviennent des titres servant à la désignation et au classement des dignitaires. Cela constitue un idiome dans lequel sont exprimés les statuts élevés des entités constituant la Grande Maison. C'est cet idiome commun à la famille du teâma et à l'organisation sociale que je voudrais analyser maintenant.

\section{Les statuts selon la formule hoor}

Chaque Maison est décrite par une séquence de la formule hoor dont l'ordre d'énonciation correspond à son statut (voir Tabl. 2.1). De façon remarquable, la séquence qui est prononcée en ouverture differe des suivantes sur deux points: Pu Doo n'est pas désignée explicitement par son nom, mais par le nom Teâ Aâôvaac qui est le nom de la Grande Maison, et il n'y a pas dans cette séquence de segment 2, décrivant les groupes importants. Les trois séquences qui suivent, débutent par le nom de la Maison qu'elles décrivent, suivi de leurs groupes importants.

Le tableau suivant (2.2) fait apparaître, horizontalement, l'ordre d'énonciation des Maisons (A à D) qui correspond à leur statut décroissant. Verticalement, sous le nom de chaque Maison, apparaissent les déterminants de son statut. Ce sont successivement la dénomination de ce statut, puis viennent les noms des paires de groupes importants - et leur 
statut -, puis les titres des paires de dignitaires - et leur statut -, suivis des indices de valorisation (T1, M1, T2, etc.) attribués à ces noms/titres selon les générations où ils apparaissent dans le système de nomination du teâma et du mweau (voir Tabl. 1).

Ce tableau fait ressortir les références systématiques aux termes teâ, mwean et $\hat{a} \hat{a} \hat{o}$ pour qualifier les statuts des Maisons, des paires de groupes importants et des paires de dignitaires (seg. 1, 2, 3) ${ }^{15}$. Il montre aussi comment les noms de la famille du teâma (T1, M1, etc., voir Tabl. 1) sont utilisés comme titres des dignitaires (dans les seg. 3). Ainsi, la première Maison

\begin{tabular}{|c|c|c|c|c|}
\hline nom de la Grande Maison & TEA AAOVAAC & & & \\
\hline $\begin{array}{l}\text { ordre d'énonciation } \\
\text { des Maisons }\end{array}$ & A & B & C & D \\
\hline $\begin{array}{l}\text { noms des Maisons } \\
\text { (seg. I) }\end{array}$ & (PU DOO)* & NOOET & TIARI & PAGANY \\
\hline leur statut & téâ & ââô & ââô & mweau \\
\hline $\begin{array}{l}\text { noms des groupes } \\
\text { importants } \\
\text { (seg. 2) }\end{array}$ & (nil) & $\frac{\text { Paabon Dui et }}{\text { Paabon Waala }}$ & $\frac{\text { Paabon Tea et }}{\text { Paabon Wiya }}$ & $\frac{\text { Paabon Gany et }}{\text { Paabon Deedan }}$ \\
\hline \multirow[t]{2}{*}{ leur statut } & (nil) & téâ & téâ & téâ \\
\hline & & mweau & mweau & mweau \\
\hline $\begin{array}{l}\text { titres des dignitaires } \\
\text { (seg. 3) }\end{array}$ & $\underline{\text { Koi }}$ et Pwanaan & Dui et Bwae & Bweon et Kaba & Gany et Damagilu \\
\hline leur statut & téâ mweau & téâ mweau & téâ mweau & téâ mweau \\
\hline $\begin{array}{l}\text { noms correspondant } \\
\text { à ce titre (Tabl. I) }\end{array}$ & $\mathrm{TI} \quad \mathrm{Ml}$ & T2 TM*** & T3 T3 & M2 NP*** \\
\hline
\end{tabular}

Tabl. 2.2 Les statuts selon la formule hoor

(Pu Doo) est dite être "sur les noms " Koi et Pwanaan, ceux du premier teâma Aâovaac et de son cadet, dans le mythe d'origine (T1 et M1). Les autres noms des teâma et mwean (T2, M2, T3, M3) ainsi que deux autres noms d'hommes de la famille du teâma (Bwae et Damagilu, TM et NP) deviennent les titres des dignitaires des trois autres Maisons, de la façon suivante. Dui (T2) est le titre du teâ de Nooet, son mweau est Bwae (TM) car Gany (M2, nom du mweau de Dui) est devenu titre du teâ de Pagany. Bweon et Kaaba (T3, M3) sont les titres des dignitaires de Tiari. La dernière Maison, Pagany, est qualifiée de mweau, statut qui coïncide avec la qualité

15. Par contraste, si la plupart des sous-clans énumérés dans les segments $4 \alpha, 4 \beta$, A $\gamma$, etc., sont eux aussi groupés par paires, ils ne sont pas, pour la plupart, en rapport tế-mweau. 
originelle de mweau de Gany (M2), nom qui cependant devient titre de têै - à qui Damagilu (NP) est adjoint comme mweau.

Ces spécificités dans la distribution des titres à Pagany sont interprétées comme témoignant de l'arrivée des groupes de cette Maison à une date postérieure à celle des trois autres Maisons. Le qualificatif de "Maison de bord de mer " (Poone mwa) attribué à Pagany, est interprété de même. Mes interlocuteurs de Paabon Dedan confirment ces faits et pointent Fidji comme leur région d'origine (en contradiction explicite avec les affirmations de Marie-Joseph Dubois 1985: 42).

Ces titres, comme les noms auxquels ils correspondent (Tabl.1), ont un statut décroissant pour les générations qui suivent celle de Koi et Pwanaan (T1 et M1), le premier teâma du mythe et son cadet. Cela rend compte du fait que la Maison Nooet, qualifiée de ââô, a un statut supérieur à celui de la Maison Tiari - elle aussi qualifiée de ââo : en effet, le teâa de Nooet est Dui (T2), nom de la deuxième génération, alors que celui de Tiari est Bweon (T3), qui appartient à la génération suivante.

Claude Lévi-Strauss fait, dans La pensée sauvage (1962 : 252), une intéressante remarque: "Du nom au titre, on passe donc par une transition insensible " liée "au rôle structural qu'ils jouent dans un système classificatoire » et - dans le cas étudié ici - statutaire. Un trait général de ce système est que les noms personnels des teâma et des mweau (et d'autres hommes de leur famille) sont utilisés comme titres des dignitaires des Maisons - ce que souligne, dans la formule hoor, l'expression "sur les noms de... »- et sont des indices de valorisation de celles-ci. Par contraste avec cette labilité de leur application comme nom ou comme titre, la stabilité de l'indice de valorisation attaché à ces signifiants est remarquable, qui les rapproche des termes de relation d'aînesse teâ, ââo et mweau. Quels en sont les principes?

\section{Les principes de valorisation}

Venons-en aux principes des différences de valorisation des statuts dans cet idiome. Ils se fondent sur deux formes distinctes de la temporalité des générations. 1) Dans une même génération, l'antériorité - temporalité qui distingue l'aînesse - est facteur de valorisation. Elle est décrite par trois termes qui par ordre décroissant de valeur sont teâ, fils aîné de teâma, ââo, fils aîné dans la famille du teâma et mweau, cadet d'un teâ. De façon périodique, génération après génération, cette temporalité de l'aînesse est réactivée avec chaque naissance (ou adoption) d'un teâ, ââô ou mweau. 2) La temporalité de la succession de trois générations. C'est celle des noms et des titres. Ici, la valorisation décroît de génération en génération, à partir des 
noms des premiers teâ et mweau. Après trois générations, cette valorisation est réactivée par un retour périodique à ces noms (T1 et M1, voir Tabl. 1).

Dans ces deux systèmes périodiques, la valorisation est associée à une antériorité. Antériorité de naissance (ou d'adoption) dans la même génération pour le système de l'aînesse. Pour le système des noms qui fonctionne sur trois générations, l'antériorité est définie en référence à l'acte de fondation de la Grande Maison décrit dans le mythe. Soulignons un point qui sera développé ultérieurement, par contraste avec les concepts de firstness de Mashall Sahlins et de precedence de James Fox : dans tous les cas, il s'agit ici d'une antériorité que je nomme "relative ", car elle est réactivée périodiquement. Et dans les deux cas, la valorisation est elle aussi réactivée de façon périodique avec la suite des générations. Cela a aussi pour effet de lier la perpétuation de la famille du teâma et celle de la société. Je montrerai plus loin qu'un autre type de temporalité, ancrée dans des mythes et valorisant aussi une forme d'antériorité "relative ", est à l'œuvre dans l'organisation sociale de la Grande Maison (voir infra, pp. 72-73).

Ainsi, sous une forme très concise, la formule hoor décrit l'organisation de la Grande Maison sous l'angle des statuts, au moyen d'un idiome fondé sur des termes spécifiques au teâma et à sa famille. Cet idiome commun établit une relation d'inter-représentation entre le teâma, sa famille et la Grande Maison. Les statuts ainsi présentés dans les discours déterminent une part significative de l'organisation des cérémonies de la Grande Maison ${ }^{16}$.

\section{Statut des Maisons et ordre de préséance cérémonielle}

Dans les cérémonies internes de la Grande Maison, les statuts induisent l'ordre de préséance, défini ici comme l'ordre d'arrivée des groupes ou de leurs représentants et celui de présentation des prestations. Cela concerne: (1) La "cérémonie des ignames" (thî̀lîn uvi, litt. : "griller l'igname»), où les dignitaires des Maisons Nooet, Tiari, Pagany et les représentants des groupes de ces Maisons se rendent chaque année à la Maison Pu Doo avec les ignames de prémices. (2) Les premiers actes de construction de la grande maison ronde, à Pu Doo. D'abord est planté le poteau central, par un sous-clan de $\mathrm{Pu}$ Doo appartenant au clan accueillant. Ce même sous-clan intronise le teâma et l'expression planter le poteau central signifie ces deux rôles, architectural et social, le teâma

16. Quelques remarques comparatives. Alors même qu'elle met en œuvre un idiome de nomination et d'aînesse, en aucun cas la formule hoor ne peut être considérée à proprement parler comme une "généalogie », terme souvent utilisé pour décrire des discours formalisés dans d'autres régions de la Grande Terre, par exemple celui qui ouvre le livre de Jean-Marie Tjibaou et Philippe Missotte (1978). On trouvera une critique de l'utilisation de ce terme "généalogie", à propos des discours viva de Kouaoua, dans Patrick Pillon 1992, notes 45, 46. Pour définir ces discours, Pillon adopte le terme de « hiérarchie statutaire » .../... 
étant lui-même qualifié de poteau central. Puis les poteaux du pourtour de la maison sont plantés par des groupes des autres Maisons, dans l'ordre de préséance. (3) La réception et l'expédition des messages formels dans les contextes concernant les relations de la Grande Maison avec d'autres Grandes Maisons. Les messages sont d'abord reçus à la Maison Pu Doo, puis redistribués selon un circuit déterminé par l'ordre de préséance : on prévient Nooet, puis Tiari, enfin Pagany. (4) Les cérémonies de paix ou de réconciliation (thaledo) concernant la Grande Maison. (5) L'intronisation du teâma. (6) Les funérailles de dignitaires: la nouvelle est portée à la Maison Pu Doo où sont prises les décisions concernant l'organisation des funérailles. La distribution des messages aux autres Grandes Maisons et aux dignitaires de chaque Maison s'effectue alors à partir de $\mathrm{Pu}$ Doo, selon l’ordre de préséance qui sera aussi celui des arrivées aux funérailles et des échanges. (7) Les mariages de personnes de statut important ${ }^{17}$. L'importance des messages montre à nouveau que ces cérémonies qu'il faut bien qualifier "d'internes» impliquent souvent des relations «externes». L'essentiel pour notre propos est que les activités cérémonielles les plus importantes de la Grande Maison sont ordonnées en fonction des statuts des Maisons, de leurs groupes importants et de leurs dignitaires. Il convient de distinguer entre les cérémonies 1 à 5, où $\mathrm{Pu}$ Doo est le lieu focal de la préséance cérémonielle - le lieu vers lequel se dirigent les prestations des autres Maisons - et les cérémonies 6 et 7 où, le plus souvent, Pu Doo est la première Maison dans la préséance cérémonielle.

Précisons que ces relations de statut ne sont pas des relations de commandement : chaque Maison, chaque groupe, a une grande autonomie pour ses affaires internes. Le teâma, l'orateur et les dignitaires des Maisons président à l'organisation des cérémonies. Le cas échéant, ils participent au règlement des litiges sur le plan de la Grande Maison. Cela n’intervient qu’en

dans l'acception courante du mot hiérarchie, différente de celle que j'utilise plus loin (voir infra, pp. 7576). Il mentionne l'importance de l'ordre de présentation (ibid. : 85), de l'imbrication de principes ternaires et binaires et du terme polysémique mwarö signifiant "maison des " mais qu'il traduit de façon restrictive par "lignage" (ibid. : 81). Selon cet auteur, les principes de l'organisation sociale "ne se laissent pas aisément circonscrire" (ibid. : 92). Jean Guiart (1992b) exprime son désaccord avec Pillon sur plusieurs points (voir Pillon 1993). J'étudie ici, pour la formule hoor, ce que Guiart (1992b : 244) nomme, pour les viva, "la structure commune à toutes [leurs] expressions successives ». Pour Houaïlou, Michel Naepels (1998: 119-120, 122) n'étudie pas les parallélismes, qu'il suggère, des discours vivaa avec les cérémonies. Il focalise son étude sur les conflits en une approche politique universalisante des relations sociales (ibid. : 18, 329). Cette problématique l'amène à évoquer l'existence de versions différentes, conflictuelles, des vivaa (ibid. : 122-123) et le fait qu'ils transmettent des savoirs sur les accueils (ibid. : 125). Un trait important des vivaa, qui les rapproche de la formule hoor et de la plupart des discours cérémoniels, est qu'ils "requièrent pour être décryptés un savoir inégalement répandu " (ibid. : 121).

17. J'ai assisté aux cérémonies $1,3,4$ et 6 . Les cérémonies 5 et 7 , et la construction de la grande maison m’ont été décrites. 
tout dernier recours, après des mois - ou des années - de discussions dans la Maison concernée et peut mener à des cérémonies de réconciliation ${ }^{18}$.

L'importance de l'ordre de préséance est mise en relief par l'attribution de maladies graves et de morts au fait que les cérémonies ou messages décrits ci-dessus ont été mal exécutés, en particulier sans respecter cet ordre. Cela signifie, bien sûr, qu'à l'occasion cet ordre peut ne pas être respecté, par ignorance, par pragmatisme (pour mettre fin à une attente très prolongée par exemple) ou par la volonté d'une personne ou d'un groupe de se mettre en avant. Les sanctions éventuelles résultent du contrôle sur la société et le monde exercé par les " anciens » et " ancêtres " (tous, vivants ou morts, nommés hulac) qui sont l'instance ultime de validation du déroulement des cérémonies ${ }^{19}$.

\section{Statuts, préséance cérémonielle et principes d'antériorité}

Les conclusions qui viennent d'être développées dérivent pour une large part de l'analyse de l'ordre d'énonciation de la formule hoor. Il convient maintenant de les compléter par celle du non-dit et de l'implicite - si on préfere, de la non-énonciation. Il importe en effet de définir plus précisément les spécificités de la Maison Pu Doo en relation avec l'ensemble de la Grande Maison et les trois autres Maisons. Car Pu Doo est assimilée à la Grande Maison dans deux acceptions de cette notion: la société prise comme unité sociale et la grande maison ronde. Pour décrire ces spécificités et en proposer une interprétation intégrant les commentaires qui m’ont été proposés par les gens d'Arama et l'analyse des échanges cérémoniels impliquant ces Maisons, on commencera par des remarques ayant trait aux formes rhétoriques d'expression du respect envers les entités sociales de statut important, on s'intéressera ensuite à leur expression dans la formule hoor, puis on reviendra à deux mythes, celui qui rend compte de l'arrivée et de l'accueil du teâma et celui qui décrit l'origine du clan accueillant.

Il existe à Arama de nombreuses manières d'exprimer le « respect» (thu phwaalu, litt. : «faire lourd») vis-à-vis des entités sociales valorisées. Celles

18. De 1992 à 1998, il y eut à Arama deux conflits ouverts et violents. Le premier, relatif à l'utilisation d'une parcelle, n'a pas fait de blessés, mais des dégâts matériels. Il a conduit à des poursuites devant la justice française qui a prononcé des peines de prison ferme. Après plusieurs mois, une première cérémonie de réconciliation a été accomplie, préalable indispensable à la participation à des funérailles des groupes concernés par le conflit. Le second conflit découlait de rivalités entre jeunes gens et a fait un blessé ; deux maisons ont été brûlées. Il a été suivi de plusieurs cérémonies de réconciliation dont la dernière, la plus importante (nommée thaledo) visait à mettre un terme définitif aux dissensions entre tous les groupes concernés. Un conflit «sourd» (expression que j'emprunte à Christine Salomon 1998), ancien, entre des groupes qui sont classiquement en opposition périphérie/centre, et sans expression violente entre 1992 et 1998, a été l'objet d'une cérémonie de réconciliation en 1997.

19. De façon générale, dans cette société le déclenchement de la plupart des maladies est attribué à des relations mal conduites avec les vivants ou/et les morts. 
qui nous concernent particulièrement dans le cadre de cet article sont la façon de désigner certains groupes, les modalités d'utilisation de certains noms propres, les formes rhétoriques de l'implicite et du non-dit. On utilise des expressions particulières pour distinguer des groupes de statut important, comme hulaya aju (litt.: "personnes ancestrales ») pour désigner la «famille du teâma " ${ }^{20}$, ou paabon (litt. : " petits-enfants») pour les groupes importants qui sont teâ ou mweau dans chaque Maison (voir supra). Si la première expression est clairement valorisante par sa référence explicite aux " ancêtres », la seconde, en revanche, procède par atténuation, et ses connotations valorisantes se comprennent en relation au système de nomination courant et à l'alternance des générations (voir note 8). Pour ce qui est de l'usage des noms, alors que ceux des Maisons Moet, Tiari et Pagany sont d'usage courant, les noms de la Maison Pu Doo et de la Grande Maison Teâ Aâôvaac sont des appellations solennelles, respectueuses, rarement utilisées dans la conversation, réservées aux discours cérémoniels. Enfin, dans la formule hoor, l'implicite et le non-dit renvoient aux relations entre la Maison $\mathrm{Pu}$ Doo, les trois autres Maisons et l'ensemble de la Grande Maison ainsi qu'aux relations entre le clan accueillant et la famille du teâma.

La formule hoor s'ouvre par une première séquence qui possède plusieurs spécificités. Elle débute par le nom de la Grande Maison, Teâ Aâôvaac (Tabl. 2, seg. 1), est dépourvue de segment 2, se poursuit «sur les noms " des premiers teâma et mweau du mythe (seg. 3), puis vient la composition de la seule Maison Pu Doo en termes de sous-clans (seg. $4 \alpha, \beta, \gamma$, etc.). Cette première séquence décrit donc la Maison $\mathrm{Pu}$ Doo, sans la désigner par son nom, et differe des trois séquences suivantes dans lesquelles les Maisons sont nommées (seg. 1) ainsi que leurs dignitaires (seg. 3) et tous leurs groupes (seg. 2 et 4). La première séquence se situe donc d'emblée, par l'énonciation du nom Teâ Aâôvaac, au niveau de l'unité de la Grande Maison, alors qu'elle décrit la seule Maison Pu Doo, c'est-à-dire une de ses parties constitutives. Deux opérations rhétoriques - substitution valorisante d'un nom pour un autre et non-dit - suscitent l'exaltation du statut de teâ de la Maison Pu Doo.

Premièrement, le nom de Pu Doo n'est pas prononcé, tandis que celui de Teâ Aâovaac l'est, qui renvoie tout à la fois à la Grande Maison dans son unité et sa totalité, et à la famille Aâôvaac, celle du teâma, qui réside à $\mathrm{Pu}$ Doo. Cette substitution du nom de la Grande Maison à celui de $\mathrm{Pu}$ Doo va de pair avec une valorisation, qui renvoie au fait que la Maison $\mathrm{Pu}$ Doo est, dans la formule hoor et dans certaines cérémonies (supra: cérémonies 1 à 5), à la fois une des quatre Maisons et, d'une certaine transformation euphonique de hulac, mot qui désigne les anciens, vivants, et les ancêtres, défunts. 
façon, que l'on précisera plus loin, représente toute la Grande Maison. Deuxièmement, cette première séquence ne comporte pas, comme les trois autres, de paire de groupes importants (seg. 2). Implicite est la relation entre deux groupes, la famille du teâma et le clan accueillant. On se souvient que, selon un mythe, ce dernier détenait le teâma précédent et a accueilli le teâma Aâovaac. Dans les faits, un de ses sous-clans intronise le teâma. L'orateur, qui scande la formule hoor, appartient à ce clan accueillant dont il n'énonce ni le nom, ni le statut important de Paabon Teâ. Dans la formule hoor, comme dans le mythe, il s'efface, avec son clan, derrière le nom Teâ Aâôvaac. Les relations du clan accueillant avec le teâma, dans le mythe et dans les faits, different de la relation aîné-cadet des groupes importants des autres Maisons (décrite dans les seg. 2 des séquences $\mathrm{B}, \mathrm{C}, \mathrm{D}$, Tabl. 2.2). La temporalité de cette relation est en effet ancrée dans un développement narratif mythique soulignant l'antériorité de l'accueillant qui installe le teâma, ainsi que le fait qu'il lui donne une épouse, ouvrant sur la constitution et la perpétuation de sa famille. L'antériorité a ici deux facettes, celle de l'accueil et celle de l'affinité.

Une autre forme d'antériorité est exprimée dans la temporalité du mythe d'origine du clan accueillant. Celui-ci raconte un départ d'une île distante, dévastée par l'épidémie et la sécheresse, et l'arrivée à Pu Doo, sur une terre déjà peuplée par des habitants Hoot qu'il repousse loin de la côte. Cela rend compte de l'appartenance Whaap de la Grande Maison d'Arama, sous la forme actuelle Teâ Aâôvaac, comme sous celle qui, selon le mythe du teâma, lui préexistait et se nommait Teâ Yhuen. (Sur le plan régional, les Grandes Maisons Hoot sont généralement considérées comme antérieures à celles qui sont Whaap.) Ainsi, le mythe du clan accueillant et celui du teâma mettent en œuvre des principes d'antériorité relative qui permettent l'accueil et orientent les relations d'affinité. Ce sont là les principes fondateurs de la Grande Maison. Ils sont aussi ceux de sa perpétuation, ou reproduction, dépendant de la suite des générations $^{21}$, conceptualisée ici autour du système périodique ternaire qui caractérise la famille du teâma et renvoie aux statuts des entités constitutives de la Grande Maison.

\section{Antériorité et organisation sociale}

Le principe d'antériorité, dont nous venons d'explorer différentes facettes, correspond jusqu'à un certain point au concept très large de firstness, proposé par Marshall Sahlins (1985: 140) pour rendre compte de la valorisation des chefs polynésiens. Ceux-ci partagent la caractéristique 
d'antériorité avec leurs dieux, ce qui contribue à les en rapprocher. La société de Mélanésie étudiée ici fonde sur le principe d'antériorité non seulement la valorisation de son personnage suprême, mais aussi celle des statuts élevés de ses entités constitutives: Maisons, dignitaires, groupes importants. J'ai souligné qu'il s'agissait à Arama d'antériorité relative. Le principe d'antériorité est donc, à Arama, à la fois plus répandu dans les relations sociales que celui mis au jour pour la Polynésie par Sahlins - où il concerne les seuls chefs et dieux - et surtout il a un caractère relatif car il est réactivé avec la suite des générations, leur renouvellement et la succession des arrivées et des accueils.

Spécialiste de l'Indonésie orientale et animateur avec ses collègues de Canberra d'un important projet austronésien ${ }^{22}$, James Fox (1994: 87) a proposé le «concept de precedence» pour «l'étude comparative des sociétés austronésiennes». Le principe d'antériorité tient une place considérable dans ce concept, avec des différence significatives en regard de celui mis en avant ici. Aboutissement de recherches portant sur des «structures d'origines" austronésiennes, deux traits principaux caractérisent la precedence. Premièrement des oppositions de paires asymétriques orientées en "valence». Deuxièmement une préoccupation concernant les origines, points d'ancrage à partir desquels se déploient des «lignes de precedence». Bien souvent, les origines sont désignées par des termes (dérivés d'une racine austronésienne identique, notée pubun, pour les langues d'Indonésie) signifiant le «début», le "tronc» et la "base d'un arbre" (Fox 1988, 1994). Plusieurs traits rapprochent la société kanak étudiée ici de ce schéma général proposé pour l'Austronésie. 1) L'importance de l'agencement des entités sociales par paires, en particulier sous la forme asymétrique aîné-cadet (teâ mweau). 2) On a noté que le nom de la Maison Pu Doo signifie "origine de la terre ", d'un terme nyelayu $p u$, signifiant « origine, commencement, tronc et base d'un arbre", renvoyant à la même racine austronésienne (notée puqun pour les langues de Nouvelle-Calédonie; Françoise Ozanne-Rivierre, communication personnelle). Enfin, 3) l'ordonnancement des cérémonies et de la formule hoor s'opère selon les principes de préséance cérémonielle. Ces parallélismes témoignent de la pertinence de ces propositions quant à une approche générale comparatiste. Mais les différences n’en présentent pas moins un intérêt considérable. D'abord, et le point est d'importance, le terme anglais precedence comme le mot français préséance signifient «droit de précéder [...] dans une hiérarchie protocolaire » et " prérogative du rang "

22. Nom appliqué dans ce projet aux sociétés qui parlent des langues de la famille austronésienne qui s'étend de Madagascar à la Polynésie (250 millions de locuteurs, plus de 1000 langues) et à laquelle appartiennent les 28 langues autochtones de Nouvelle-Calédonie. Le champ d'étude est ici constitué linguistiquement (Fox 1994 : 96). La pertinence de l'équation langue=société a été questionnée par Joseph Platenkamp (1990). 
(Le Robert). Toutefois, le sens premier du mot anglais est «antécédent » et "le fait de précéder dans le temps" (Webster). Fox utilise ce terme principalement dans ce sens premier et lui donne un caractère quasi historique. Conformes au sens premier du mot anglais precedence, les propositions de Fox se concentrent sur des "lignes de precedence [qui doivent] nécessairement avoir un initium ou un point d'origine» (initial term or inception point; Fox 1994 : 99). Cela suggère une temporalité linéaire ancrée sur un point fixe et donc une antériorité absolue ( nous sommes les premiers arrivants ") : la precedence ne fait pas la distinction entre première occupation d'un lieu et préséance cérémonielle.

Comme la precedence est "socialement un important sujet de contestation, [et] est ouverte à des processus de réorganisation complexes " (Fox, ibid. : 98), il existe des inversions dans les différentes versions de la precedence données par divers groupes. Les analyses d'exemples ethnographiques indonésiens - Timor, Roti, Java - faites par Fox privilégient ce temps linéaire ancré sur une origine fixe. Le constraste est donc considérable avec Arama, où la préséance cérémonielle se fonde, comme l'ensemble du système présenté ici, sur le principe d'antériorité relative. De 1992 à 1998 des affirmations divergentes pouvaient, à l'occasion, porter sur l'antériorité de la présence à Arama de certains sous-clans par rapport à d'autres, ou même par rapport à des groupes importants ou au groupe accueillant ("nous étions arrivés avant eux»). Mais elles n’étaient pas développées de façon conflictuelle ouverte par les groupes concernés et, surtout, ne mettaient pas en cause la valorisation relative des Maisons, des groupes importants et des dignitaires dans l'ordre de préséance lors des cérémonies ou des discours hoor. Car les principes d'antériorité relative qui commandent le statut sont largement indépendants des affirmations sur l'antériorité d'installation - y compris, je voudrais y insister, chez le clan accueillant. Le statut est largement disjoint de l'ordre d'arrivée. L'exemple d'Arama suggère que le "concept de precedence " gagnerait à prendre en compte de façon plus fine la conceptualisation sociale des temporalités.

Le troisième outil anthropologique que je voudrais confronter à l'organisation de cette Grande Maison est la notion de hiérachie, dans le sens que lui a donné Louis Dumont, à partir de ses travaux sur le système indien des castes (Dumont 1966, 1983). Pour cela, il importe de poursuivre l'analyse de l'organisation de la Grande Maison et de ses cérémonies.

Dans les cérémonies régionales, la formule hoor présente la Maison $\mathrm{Pu}$ Doo comme implicitement assimilée à la totalité de Teâ Aâovaac mais aussi comme étant une partie de cette totalité, une de ses quatre Maisons constitutives. Dans les cérémonies internes de la Grande Maison, Pu Doo est soit le point focal où convergent les délégations et prestations des trois 
autres Maisons (cérémonies 1 à 5), soit la première des quatre Maisons (cérémonies 6 et 7). Dans cette perspective, on peut résumer l'ensemble des relations de la Grande Maison comme suit:

I. Une relation d'englobement des quatre Maisons par la Grande Maison Teâ Aâovaac. C'est le niveau supérieur du système, celui de l'unité de la Grande Maison ${ }^{23}$, figuré dans l'architecture de la grande maison.

IIa. La Maison Pu Doo entretient une relation privilégiée avec ce niveau supérieur, dans laquelle elle est assimilée à la Grande Maison, ce qui s'exprime par la relation spatiale et temporelle de focalisation dans les cérémonies internes ( 1 à 5 ) où les trois autres Maisons se rendent à Pu Doo. Dans les relations régionales, cela est exprimé par les spécificités internes de la première séquence de la formule hoor.

IIb. Pu Doo a aussi, vis-à-vis des trois autres Maisons, une relation temporelle d'antériorité dans l'ordre de préséance qui se manifeste dans d'autres cérémonies internes $(6,7)$ et, dans les relations régionales, par sa première place dans l'ordre d'énonciation des séquences de la formule hoor.

III. Les trois autres Maisons sont dans une relation en cascade, selon leur ordre décroissant de statut : Nooet, Tiari, Pagany.

Ainsi, paradoxalement, la Maison $\mathrm{Pu}$ Doo est à la fois une figure de l'unité et de la totalité de la Grande Maison et une partie - éminente parmi les quatre qui la constituent. La Maison $\mathrm{Pu}$ Doo est donc en relation à deux niveaux, celui de la Grande Maison - d'un certain point de vue, $\mathrm{Pu}$ Doo est la Grande Maison - et le niveau de ses parties constitutives - d'un autre point de vue, Pu Doo est la première des quatre Maisons. Cet « englobement du contraire» est central au concept de hiérarchie proposé par Dumont. Celui-ci a défini la «relation hiérarchique élémentaire [... comme] celle entre un tout (ou un ensemble) et un élément de ce tout (ou ensemble), ou encore entre deux parties par référence au tout; elle s'analyse en deux aspects contradictoires de niveau différent : distinction à l'intérieur d'une identité, englobement du contraire. La hiérarchie est ainsi bidimensionnelle» (Dumont 1983 : 263). À Arama le niveau global (cidessus : I) est la Grande Maison, ensemble ordonné de quatre Maisons qui forme une unité. Dans la description de l'organisation de cette Grande Maison et dans son actualisation cérémonielle, $\mathrm{Pu}$ Doo, qui est une des quatre Maisons constituant la Grande Maison est cependant à un niveau (IIa et IIb) différent de celui (III) des trois autres Maisons. Pour Pu Doo, la relation privilégiée à la figure unitaire englobante de la Grande Maison

23. Il s'agit d'une unité de relations : la Grande Maison Teâ Aâôvaac étant elle-même un sous-système dans le réseau de relations Hoot ma Whaap, ce qu'exprime le contexte régional d'énonciation de la formule hoor (voir supra, p. 66), ainsi que l'imbrication des relations externes et internes et l'importance des figures du Chemin et de la Porte dans les relations régionales (Monnerie, à paraître). 
et la relation de focalisation cérémonielle (IIa) sont étroitement associées à l'antériorité dans l'ordre de préséance (IIb). Il existe sur $\mathrm{Pu}$ Doo deux points de vue : celui de l'unité de la Grande Maison Teâ Aâôvaac et celui de la gradation des quatre Maisons qui la constituent. Le fait que la résidence de la famille du teâma et la grande maison (quand elle existe) soient à $\mathrm{Pu}$ Doo est, à cet égard, fondamental. En effet, bien qu'étant une partie de la Grande Maison, dans certaines cérémonies la Maison Pu Doo est le point focal vers lequel converge et d'où part la circulation des prestations et des messages. Il s'opère ici ce que Dumont nomme un «changement de niveau ", où Pu Doo vient figurer la Grande Maison - le niveau global de cette société locale. Cette position de $\mathrm{Pu}$ Doo en relation avec l'ensemble de la Grande Maison et avec les parties qui la constituent, les Maisons, rapproche la forme de la hiérarchie de statut de cette société de celle de la hiérarchie de valeur proposée par Dumont ${ }^{24}$. Toutefois, ici, le principe de valorisation des statuts de la Grande Maison établit une différence avec les propositions de cet auteur pour qui dans le système des castes de l'Inde, la valeur repose sur un axe allant du pur à l'impur. Dans le système étudié ici la valorisation se fonde sur le principe d'antériorité relative.

Les sociétés kanak de l'extrême nord de la Grande Terre ont à leur tête un personnage nommé teâma "aîné suprême", qui à Arama, entretient une relation d'inter-représentation avec la société conçue comme une Grande Maison. En effet, l'idiome d'aînesse et de nomination de la famille du teâma définit la hiérarchie de statut des quatre Maisons, des groupes et des dignitaires qui constituent la société. Cela s'exprime dans les cérémonies internes de la Grande Maison ainsi que dans les relations externes de la région Hoot ma Whaap. Alors qu'en français les gens d'Arama expliquent volontiers ce système en recourant à des mots tels que « roi, prince, duc, comte", les principes qui sont à l'œuvre, tels qu'ils ressortent d'une analyse croisée des faits de parole - dans leur langue - et des actes sociaux sont, nous venons de le voir, différents de ceux de l'Ancien Régime et de la noblesse française. Cette comparaison a cependant le mérite d'appeler l'attention sur un contraste significatif dans les modalités de représentation de la société qui sont celles du roi et du teâma. Dans certaines royautés d'Europe (Kantorowicz 1989 ; Giesey 1987) le corps du souverain était

24. Dans une visée comparatiste, la relation hiérarchique a été appliquée à la description de plusieurs sociétés (Dumont 1983, 1992 ; Jamous 1981; Barraud, Coppet, Iteanu \& Jamous 1984 ; Coppet 1992). On sait que les interprétations structuralistes de ce type de problème où « le tout est dans la partie et la partie équivaut au tout par permutation" (Henri Lefebvre) sont placées sous le signe de la métonymie. Lefebvre est cité par le linguiste Marc Bonhomme (1987: 10-21) qui analyse l'influence de Roman Jakobson dans l'extension considérable qui a été donnée à l'idée de métonymie par la sémiotique et le structuralisme. 
central dans le dispositif de représentation (Coppet 1992) dérivé de celui du corps du Christ. Cela perdure dans une "fiction [...] "organiciste" ", celle de la "personne morale » qui préside à la représentation de la société, "on fait comme si les citoyens étaient les "membres" d'une personne immortelle" (Descombes 1996 : 152). Dans le système kanak d'Arama, des principes différents informent les relations d'inter-représentation de la société et du teâma. Ils dépassent en effet son seul corps, et même sa seule personne pour mettre en avant la figure de la paire asymétrique qu'il forme avec son cadet. Notons cependant que dans les deux configurations, il existe une préoccupation commune : assurer, au-delà de la mort de tel ou tel souverain, ou teâma, la perpétuation d'une forme de la prééminence sociale assimilée à un aspect de la société.

Ici toutes les différences de statuts entre les Maisons, leurs dignitaires et groupes importants sont exprimées dans un idiome dérivé du système de l'aînesse et de la nomination du teâma et de sa famille. Cela constitue une forme d'auto-référence, où l'organisation de la société renvoie aux relations constitutives du personnage du teâma et de sa famille qui, en retour renvoient à l'organisation de la société. Dans cet idiome où se combinent principes binaires et ternaires, la valorisation est fondée sur des formes différentes de l'antériorité exprimées dans des mythes ou dans la suite des générations. Dans la même génération, l'antériorité prend la forme de la relation aîné-cadet, et valorise l'aînesse. La valorisation de l'antériorité sur trois générations caractérise le système de nomination du teâma et de son cadet où la valeur la plus élevée est accordée au nom de cette paire originelle dans le mythe. Il s'agit donc, dans ces deux cas, mais de deux façons différentes, d'une antériorité sous-tendue par la suite des naissances (ou des adoptions) et des générations. Cette antériorité n'est ni absolue ni inscrite dans une temporalité linéaire, mais relative, car indexée sur la succession des générations, qui est périodiquement renouvelée. Ces phénomènes sont centraux pour la reconduction ou, si on préfere, la reproduction de la société sous sa forme de Grande Maison.

L'expression principale de ces différences de statut est aujourd'hui l'ordre de préséance cérémonielle - l'ordre des arrivées et des prestations lors des cérémonies. Ces actes se déroulent dans une temporalité parallèle à celle de l'énonciation de la formule hoor - présentant la Grande Maison dans les cérémonies régionales - qui est celle de l'ordre décroissant de statut des Maisons. On peut ainsi rapprocher la temporalité des actes cérémoniels de celle de l'énonciation de cette formule. Alors même qu'ils les lient en tant que Grande Maison, simultanément, cérémonies et discours expriment et distinguent, dans leur déroulement interne, les identités sociales et les statuts des parties de Teâ Aâôvaac. L'étude des conceptions et représentations 
de cette société concernant l'expression de la temporalité25, principalement dans les domaines des générations, des mythes, des cérémonies et des discours - et leur mise en perspective - se révèle indispensable à une compréhension fine de ces interactions systématiques entre statut et antériorité. À mon sens, la faiblesse des propositions concernant la precedence tient au fait qu'elles tendent à privilégier, pour toutes les sociétés prises en considération, un vecteur temporel orienté du passé vers le présent - dérivé de la temporalité historique que nous valorisons -, ce qui amène à considérer comme des contradictions les affirmations qui s'en éloignent. Par contraste, j’ai montré que c'est selon des principes d'antériorité relative, qui se développent autour de la dynamique des générations (conceptualisée de façon périodique) et de l'affinité, qu'est construit, dans cette société kanak, un idiome répondant d'une part considérable du déroulement des cérémonies et discours cérémoniels, de la succession des personnages suprêmes, et de certaines des continuités et discontinuités dans le temps long. Cet idiome vise à affirmer l'emprise de la société sur ses statuts, son territoire et ses temporalités, en particulier à travers ce que nous nommons le rituel et l'histoire.

C'est en définitive un double dispositif d'inter-représentation qui est ici mis en œuvre, liant la société locale avec d'une part la figure de la Grande Maison et d'autre part le teâma - et sa famille. Le premier dispositif - de représentation des sociétés locales comme Grande Maison - renvoie à des conceptions régionales qui sont aussi répandues dans le monde kanak, audelà de Hoot ma Whaap. Le second définit l'entité localisée spécifique Teâ Aâôvaac en relation avec le teâma lui-même conceptualisé comme poteau central et flèche faîtière de la Grande Maison, donc comme partie de celleci aérienne et fichée dans le sol. Cette figure renvoie à la fois à la relation de mariage du teâma du mythe avec une fille du clan accueillant et au fait que celui-ci est seul habilité à "planter » le teâma, c'est-à-dire à l'introniser. La notion de "Maison ", comme aspect de la société ou des groupes qui la constituent est attestée en Indonésie, en Mélanésie, en Micronésie et en Polynésie. Et bien que la configuration d'Arama soit originale, nombre de ses traits sont familiers aux connaisseurs de l'Océanie et de l'Indonésie : tout particulièrement l'importance, sur le plan de l'organisation de la société, de la relation aîné-cadet et des titres. C'est pourquoi les réflexions comparatives sur le thème de la Maison et sur celui des principes d'organisation et de représentation des sociétés nous entraînent au-delà de la seule Nouvelle-Calédonie.

Trois outils anthropologiques ont été confrontés à la configuration de Grande Maison étudiée ici. Aucun d'entre eux n'a de valeur universelle, en chronisation, sont au principe des activités rituelles et des expressions de la temporalité, voir Iteanu 1999. 
cela qu'aucun ne rend compte parfaitement des phénomènes observés. J'aimerais souligner, en guise d'hommage, que tous ces outils se situent dans la lignée des travaux de Arthur M. Hocart (Dumont 1966: 45 ; Fox 1994 : 107; Sahlins 1985: Introduction). La proposition de Sahlins pour la Polynésie concernant l'importance du principe de firstness assez proche de l'idée d'antériorité, s'étend en fait à des sociétés de Mélanésie, celle d'Arama, mais aussi certaines sociétés de Fidji et des Salomon (Hocart 1952; Monnerie 1998). Le modèle de la precedence élaboré par Fox possède une pertinence indéniable sur un plan général austronésien, c'est un outil comparatif qui met en regard l'Indonésie et l'Océanie, apportant ainsi à nos études un décloisonnement salutaire. Initialement élaboré à partir de l'Inde, le modèle de la hiérarchie de valeur de Dumont relève, lui, un autre défi comparatiste: rendre compte de certains phénomènes sociaux "paradoxaux » du point de vue de notre sens commun, ce qui est le cas de la position complexe de la Maison Pu Doo dans la Grande Maison. Par une approche qui porte en elle les deux formes - pragmatique et théorique - de la rationalité occidentale, ces outils anthropologiques soumettent «à la critique nos croyances les plus chères " (Popper 1998) et permettent d'aller plus avant dans la comparaison et la compréhension des sociétés.

MOTS CLÉS/KEYWORDS: représentation/representation - maison/house - nomination/name giving - temporalitéltime - Océanie/Oceania - Nouvelle-Calédonie/New Caledonia. 


\section{Ammann, Raymond}

1997 Danses et musiques kanak. Nouméa, Agence de développement de la culture kanak.

Barraud, Cécile, Daniel de Coppet, André Iteanu \& Raymond Jamous

1984 «Des relations et des morts. Quatre sociétés vues sous l'angle des échanges ", in Jean-Claude Galey, ed., Différences, valeurs, hiérarchie. Paris. Éditions de l'École des hautes études en sciences sociales : 421-520.

Bensa, Alban \& Jean-Claude Rivierre

1982 Les chemins de l'alliance. Paris, SELAF.

Bonhomme, Marc

1987 Linguistique de la métonymie. Paris, Peter Lang.

\section{Boulay, Roger}

1990 La maison kanak. Contributions de Alban Bensa et Alain Saussol. Marseille, Parenthèses/[Nouméa], ADCK/Paris, Orstom.

\section{Coppet, Daniel de}

1992 "Comparison, A Universal for Anthropology ", in Adam Kuper, ed., Conceptualizing Societies. London, Routledge: 59-74.

Descombes, Vincent

1996 Les institutions du sens. Paris, Éditions de Minuit.

\section{Douglas, Bronwen}

1970 «A History of the Balade People of New Caledonia, 1774-1845», Journal of the Polynesian Society (Wellington) 79 (2) : 180-200.

Dubois, Marie-Joseph

1985 Histoire résumée de Belep (NouvelleCalédonie). Nouméa, Société d'études historiques de la Nouvelle-Calédonie («Publications » 37).

\section{Dumont, Louis}

1966 Homo hierarchicus. Essai sur le système des castes. Paris, Gallimard ("Bibliothèque des sciences humaines").

1983 Essais sur l'individualisme. Paris, Le Seuil.

1992 "Anthropologie, totalité et hiérarchie», in Philosophie et anthropologie. Paris, Éditions du Centre Georges Pompidou: 11-24.

Fox, James

1988 "Origin, Descent and Precedence in the Study of Austronesian Societies ", in Lecture in Connection with De Wisselleerstoel Indonesische Studies. Leiden, The

Netherlands.

1994 "Reflections on "Hierarchy" and "Precedence" ", History and Anthropology 7 (1-4) : 87-108.

Front national de libération kanak socialiste (FNLKS)

1988 Kanak. Un peuple, une culture, une lutte. Brochure collective, préface de Jean-Marie Tjibaou. Rennes, FNLKS.

Garnier, Jules

1978 Voyage à la Nouvelle-Calédonie (facsimilé d'articles de la revue Tour du Monde, 1867-1868). Nouméa, Les Éditions du Cagou.

\section{Giesey, Ralph}

1987 Le roi ne meurt jamais. Les obsèques royales dans la France de la Renaissance. Paris, Flammarion.

\section{Godin, Patrice}

1990 "Maisons, chemins et autels", in De jade et de nacre. Catalogue d'exposition. Paris, Réunion des musées nationaux.

\section{Guiart, Jean}

1966 Mythologie du masque en NouvelleCalédonie. Paris, Société des océanistes («Publications »). 
1992a Structure de la chefferie en Mélanésie du sud, vol. I. Paris, Musée de l'Homme-

Institut d'ethnologie. 1992b «À propos de la vallée de

Kouaoua ", Journal de la Société des

Océanistes 95 : 241-249.

Hocart, Arthur M.

1952 The Northern States of Fiji. London, Royal Anthropological Institute of Great Britain and Ireland ( Occasional

Publication" 11).

Iteanu, André

1999 "Synchronisations among the Orakaiva ", Social Anthropology 7 (3) : 265-278.

Jamous, Raymond

1981 Honneur et baraka. Les structures sociales traditionnelles dans le Rif. Paris, Éditions de la Maison des sciences de l'homme/ Cambridge, Cambridge University Press.

\section{Kantorowicz, Ernst}

1989 Les Deux Corps du Roi. Paris, Gallimard (1 ${ }^{\text {re }}$ éd. 1957).

Kling, Georges

1989 L'Alcmène. L'exploration

de la Nouvelle-Calédonie, 1848-1851.

Nouméa, Société d'études historiques de la Nouvelle-Calédonie ("Bulletin» 80).

Lambert, Père

1900 Mours et superstitions

des Néo-Calédoniens. Nouméa, Nouvelle

Imprimerie nouméenne.

Leenhardt, Maurice

1980 Notes d'ethnologie néo-calédonienne.

Paris, Musée de l'Homme, Institut d'ethno-

logie ( $1^{\text {re éd. } 1930)}$

Lévi-Strauss, Claude

1962 La pensée sauvage. Paris, Plon.

Monnerie, Denis

1996 Nitu. Les vivants, les morts et le cosmos selon la société de Mono-Alu (îles Salomon). Leiden, Center for Non Western Studies. 1997 «Identités sociales et relations régionales en Océanie (îles Salomon du Nord-
Ouest) ", in Joël Bonnemaison, Luc

Cambrézy, Laurence Quinty-Bourgeois, eds, CD ROM. Paris, Orstom Éditions.

1998 «Oceanic Comparison Reconsidered.

The Mono-Alu Problem », Social

Anthropology 6 (1) : 91-107.

à paraître La parole de notre Maison.

Mortelier, Christiane

1993 «Une escale à la mission d'Arama en

1863. Le Dr Louis Thiercelin et son journal d'un baleinier ", Bulletin de la Société

d'études historiques de Nouvelle-Calédonie $94: 12-47$.

Naepels, Michel

1998 Histoires de terres kanakes. Paris, Belin.

Ozanne-Rivierre, Françoise, avec la collab. de Baptiste Boiguivie, Scholastique Boiguivie \&

Éliane Dedane

1998 Le nyelâyu de Balade (Nouvelle-

Calédonie). Paris, Peeters.

Pillon, Patrick

1992 "Listes déclamatoires ("viva”) et principes d'organisation sociale dans la vallée de la Kouaoua (Nouvelle-Calédonie) ", Journal de la Société des Océanistes 94 : 81-101.

1993 « Kouaoua et la méthode de Jean Guiart en anthropologie ", Journal de la Société des Océanistes 96 : 83-89.

Platenkamp, Joseph

1990 North Halmahera: Non-Austronesian Languages, Austronesian Cultures?, Leiden, Het Oosters Genootsschap (Netherlands).

Popper, Karl

1998 Des sources de la connaissance et de l'ignorance. Paris, Payot Rivages ( $1^{\mathrm{re}}$ éd. 1963).

\section{Rozier, Claude}

1990 La Nouvelle-Calédonie ancienne. Paris, Fayard.

Sahlins, Marshall

1963 «Poor Man, Rich Man, Big Man, Chief: Political Types in Melanesia and 
Polynesia ", Comparative Studies in Society

and History 5 : 285-303.

1985 Islands of History. Chicago, The

University of Chicago Press.

\section{Salomon, Christine}

1998 «La personne et le genre au Centre

Nord de la Grande Terre (Nouvelle-

Calédonie)», Gradhiva 23:81-100.
Thomas, Nicholas

1989a «The Force of Ethnology : Origins

and Significance of the Melanesia/Polynesia

Division ", Current Anthropology 30 (1) : 27-41.

1989b "Reply to Comments ", Current

Anthropology 30 (2) : 211-213.

Tjibaou, Jean-Marie \& Philippe Missotte

1978 Kanaké. Papeete, Éditions du Pacifique.

\section{RÉSUMÉ/ABSTRACT}

Denis Monnerie, Représentations de la société, statuts et temporalités à Arama (NouvelleCalédonie). - À Arama, au nord de la Grande Terre, la société est conçue comme une Grande Maison, constituée de quatre hameaux nommés Maisons. Le système de l'aînesse et de la nomination du personnage suprême de cette Grande Maison fonde un idiome appliqué aux représentations de son organisation sociale et cérémonielle. Cet idiome, qui combine des principes binaires et ternaires met en œuvre des principes temporels périodiques articulés sur des générations. Il établit des différences de statuts - entre les Maisons, leurs dignitaires et certains de leurs groupes de parenté - qui valorisent différentes formes d'antériorité dont je montre qu'elles sont relatives. Des propositions de Marshall Sahlins, James Fox et Louis Dumont sont confrontées à ce système qui présente la particularité de développer les représentations de cette société d'Océanie non pas à partir d'une réflexion sur le corps de son souverain, mais à travers la terminologie de l'aînesse et de la nomination de son personnage suprême.
Denis Monnerie, Conceptions of Society, Status, and Time in Arama (New Caledonia). - In Arama, at the northern tip of Caledonia, society is thought to be a Great House made up of four hamlets, each called a House. The system of seniority and the system for naming the head of this Great House underlie an idiom that applies to its social and ceremonial organization. Combining binary and ternary principles, this idiom is grounded in principles of periodicity linked to generations. It establishes status differences (between Houses, their dignitaries and certain kin groups), which place value on forms of antecedence, that are shown to be relative. The anthropological concepts proposed by Marshall Sahlins, James Fox and Louis Dumont are tested against this original system in Oceania, based as it is on elder/junior terminology and on the way of giving a name to the head of the Great House. This contrasts with many other societies that work out their ideas of society around notions pertaining to the sovereign's body. 